significant physical and emotional demands of palliative care. Consequently, hospices employ a range of strategies to support and develop their staff, but it is not clear which are the most helpful, the best value or whether certain combinations are needed. Though ethical considerations are a prominent and common source of distress for hospice staff, clinical ethics rarely receives as explicit or frequent attention within hospices when compared to other support structures.

Methods We critically reviewed the staff support and development structures at a large hospice in southeast England, spanning: informal colleague support, reflective practice sessions, teaching, line management and specific additional support such as clinical supervision, Clinical Ethics Committee (CEC) and Schwartz Rounds.

Results Our review identified, then compared and contrasted, the key mechanisms of staff support and development: - CEC (what is the 'right thing' to do) - Reflective Practice (how to best go about doing the 'right thing') - Clinical Supervision (being personally able to do the 'right thing') - Schwartz Rounds (how doing the 'right thing' feels).

Conclusion Appropriate staff support and development is vital for hospices to deliver high quality, sensitive and individualised patient care. Four mechanisms appeared pivotal in their ability to support and develop staff in their professional roles. Despite clear overlaps, appreciation of their differences was fundamental in understanding their collective benefit: - CECs: being ethical - Reflective practice: being organised - Clinical supervision: being educated - Schwartz Rounds: being human. Subsequently, we propose the 'Support of Care Cycle' where each element informs the next in a virtuous spiral. As interdependent, to miss any component would undermine a hospice's staff support and development structures. Thus, hospices need to provide all four of these cornerstones, including the often neglected clinical ethics.

\section{INVOLVING PEOPLE WITH DEMENTIA WHO LACK CAPACITY IN RESEARCH}

J Hopkinson, A King, K Hydon, L Young, C Howells, R Tope, S Muthukrishnan. Cardiff University

\subsection{6/bmjspcare-2018-ASPabstracts. 123}

Background Empowering people with dementia to be involved in research at all stages of the disease is, respectful of individuals, maintains personhood and is equitable. It is therefore consistent with best practice in dementia care. Research is important for improving dementia treatments, care and service delivery. However, involving people with dementia who lack capacity or who have fluctuating capacity in research is challenging and requires careful consideration to avoid potential harms.

Methods This paper reports a novel and successful method for recruitment into two research studies of people with dementia. The first study investigated cancer services provided for people with dementia who received cancer treatment in 2014/ 2015. The second study investigated crisis intervention in the community for people with dementia and their family members in 2017. The coproduced approach has enabled people with dementia in primary and secondary care receiving services in their own homes, out-patient clinics and in hospitals to take part in research.

Results This paper will discuss how the researchers:
- Devised method that accommodated the needs of people with dementia for obtaining formal written consent or support for participation from a Consultee, as required by the Mental Health Act 2005.

- Negotiated the challenges of ethical and other approvals.

- Developed training for research assistants in the assessment of mental capacity and recruitment of people with dementia.

The presentation will include a flow diagram of the recruitment process. Other researchers should then be able to adopt or adapt the approach for studies involving people with dementia.

Conclusions There are challenges to involving people with dementia in research, but they can be overcome. What our research teams have learnt has informed the recently launched 'Research and impaired mental capacity in adults: guidance for researchers' published by Healthcare Research Wales. ${ }^{1}$

\section{REFERENCE}

1. https://www.healthandcareresearch.gov.wales/uploads/News/research_and_impaired_mental_capacity_in_adults-guidance_for_researchers.pdf

\section{REVIEW OF DATA FROM THE 2016 OFFICIAL REPORTS OF THE DUTCH TERMINATION OF LIFE ON REQUEST AND ASSISTED SUICIDE ACT AND OREGONS DEATH WITH DIGNITY ACT}

Baroness Ilora Finlay. House of Lords

\subsection{6/bmjspcare-2018-ASPabstracts. 124}

Of 6091 notified cases of physician assisted suicide (PAS)/ euthanasia (PAE) in the Netherlands, 3840 (63\%) were 70 years or over: the proportion rises to $86 \%$ (5248 PAS/PAE deaths) if over-60s are included.

Oregon's official report on their PAS law, which requires a prognosis of $<6$ months, shows $71 \%$ of PAS deaths in 2016 were aged 65 or over (median 73 years).

Both legislatures show rising incidences of PAS/PAE since legislation came into force.

In the Netherlands the annual numbers of deaths initially remained stable and fears of rising death rates were thought groundless. After 2007, the annual numbers of deaths began to rise steeply. In 2016, 1 in every 25 deaths the result of legalised PAS or PAE. A law like Holland's 2001 Act would probably result in around 21000 such deaths annually in England and Wales.

In 2010, of 3,136 Dutch PAS/PAE notified, 2781 (89\%) were related to cancer, cardiovascular and neurological disorders and 11 per cent to other conditions. By 2016 a rising proportion $(17 \%)$ related to multiple geriatric syndromes, dementia $(n=141)$, psychiatric disorders $(n=60)$, and other conditions. Statistics Netherlands data confirms this trend.

Extension of euthanasia caused psychiatrist Boudewijn Chabot, whose prosecution preceded Dutch legislation, to express concern, writing that the foundation of the law has been gradually eroded so that now it 'does not provide protection to people with dementia and psychiatric problems.

Non-assisted suicide rates have not fallen where PAS/PAE is legalised, but the introduction of PAS seemingly induces more self-inflicted deaths than it inhibits. WHO data shows higherthan-average suicide rates per 100000 population (2015) of 20.5 in Belgium, 15.1 in Switzerland, 12.3 in Canada, 11.9 in 
the Netherlands and 11.1 in Luxembourg - global average was 10.7 ; UK was 8.5 .

\section{Global palliative care | Posters 98-100}

\section{PALLIATIVE AND ADVANCE CARE PLANNING DISCUSSIONS WITH COPD PATIENTS}

Nuno Tavares, Nikki Jarrett, Katherine Hunt, Tom Wilkinson. University of Southampton, Solent NHS Trust and University Southampton Hospital NHS Foundation Trust

\subsection{6/bmjspcare-2018-ASPabstracts. 125}

Background Chronic obstructive pulmonary disease (COPD) is a disease of the lungs that is characterised by a consistent airflow obstruction. It is associated with persistent, progressive and irreversible breathlessness. COPD remains the fifth most common cause of death in England and Wales, accounting for about 25000 deaths a year. Despite this, only $30 \%$ of patients with COPD have had a discussion about palliative care with their clinician. When conversations do take place, they usually happen during a hospital admission when patients are very unwell and are unable to fully express their preferences, and with clinicians who do not know the patients. Furthermore, recent research has shown that clinicians do not know when and how to start and conduct conversations with COPD patients.

Aim To explore COPD patients' preferences for the timing and nature of palliative care conversations with healthcare professionals.

Methods Interviews and case-note review of COPD patients. Patients were recruited into 3 different groups according to their disease severity: mild, moderate and severe. A total of 33 patients were recruited into the study and the topics discussed during the interviews included: understanding about COPD and palliative care, care preferences and preferences for palliative and advance care planning discussions with doctors and nurses. Patients were recruited from GP practices and respiratory outpatients' clinics in the south of England from April to September 2017. The methodology for data analysis encompasses interpretative phenomenology analysis.

Results Preliminary themes of the study include: healthcare professional discussing palliative care; timing for palliative care discussions; approaches to discuss palliative care; patients" understanding about COPD; patients' understanding about palliative care; patients` care preferences.

Conclusion Greater understanding of patients' preferences and understanding of palliative care and end of life conversations will be discussed.

\section{COMPLEXITY AND OUTCOMES OF PAEDIATRIC PATIENTS CARED FOR BY A GENERAL COMMUNITY PALLIATIVE CARE TEAM}

M Doherty, F Cranfield, S McLean. St. Francis Hospice, Dublin and Department of Palliative Care, Beaumont Hospital, Dublin

\subsection{6/bmispcare-2018-ASPabstracts. 126}

Background Despite development of specialist palliative care (SPC) services for paediatric patients (PPts) in Ireland, general community palliative care teams (CPCTs) provide a significant amount of palliative care to PPts at home. Outcomes describe the change in health status arising from healthcare interventions. Measuring outcomes helps evaluate clinical care, informs resource allocation, and drives quality improvement. In one CPCT, point of care collection of clinical and workload data has been routine since 2013. Data collected is used for clinical handover, measuring workload and complexity, and outcomes measurement.

Aims and objectives To describe the complexity, workload and outcomes of PPts under CPCT; to demonstrate the feasibility of standardised outcomes measurement in SPC.

Methods Validated assessments are performed at each review:

Phase of illness;

Problem Severity Score (PSS);

Palliative Performance Scale (PPS);

Visit duration;

Healthcare professional (HCP) stress.

Data collected between 2013 and May 2017 were analysed using descriptive and analytical statistics.

Findings/results For 201 episodes (17 PPts), the median duration of care was 7 days (range 1-350). 89.1\% episodes were nursing visits; $95.2 \%$ at home. The care of PPts was more complex than of adult patients (APts). 43.4\% of episodes were in stable phase. There was no difference in mean PSS between PPts and APts (4.59 vs 4.68/12). Caregivers of PPts had higher psychosocial symptom severity compared to caregivers of APts (1.66/3 vs 0.97/3). HCPs reported stress more frequently at visits to PPts than APts $(26.4 \%$ vs $4.64 \%$ of visits). Higher HCP stress was moderately correlated with higher PSS (Pearson's $r=0.347, \quad p=0.001$ ), caregiver psychosocial issues $(\mathrm{r}=0.282, \mathrm{p}=0.001)$, and strongly correlated with patient psychosocial issues $(\mathrm{r}=0.392, \mathrm{p}<0.001)$.

Conclusions PPts under the care of CPCTs are complex, and their care may present a challenge to general CPCts. The care of PPtx is associated with significant HCP stress. Routine outcomes measurement in CPC is feasible and useful.

\section{INTEGRATING PALLIATIVE CARE; IMPACT OF A 5 YEAR STRATEGY FROM THE MAKERERE AND MULAGO PALLIATIVE CARE UNIT}

M Leng, G Kivumbi, L Grant, SM Murray, J Downing, J Turyahikyio, P Bagasha, L Namukwaya. Makerere Palliative Care Unit Department of Medicine Makerere University Kampala, Palliative care Education and Research Consortium Uganda, University of Edinburgh, Cairdeas International Palliative Care Trust UK, International Children's Palliative Care Network

\subsection{6/bmjspcare-2018-ASPabstracts. 127}

Introduction Makerere and Mulago Palliative Care Unit (MPCU) provides quality, evidence-based palliative care (PC) for patients and families through a centre of excellence delivering: an integrated clinical service within the national referral hospitals site; carrying out research, training and capacitybuilding in collaboration with partners; and developing leaders in PC.

Methods An evaluation of the 2011-2016 strategic plan was undertaken. A review of indicators was completed including: care provision; training numbers; collation of impact assessments; an online survey and qualitative interviews addressing research capacity, and the qualifications attained by MPCU staff.

Results A total of 9858 patients seen (3189 directly by MPCU with 6669 by link nurses. 4630 individuals trained including undergraduates, postgraduate, and in-service short courses. 